This item was submitted to Loughborough's Research Repository by the author.

Items in Figshare are protected by copyright, with all rights reserved, unless otherwise indicated.

\title{
Role of saddle tori in the mutual synchronization of periodic oscillations
}

PLEASE CITE THE PUBLISHED VERSION

PUBLISHER

(C) American Physical Society

LICENCE

CC BY-NC-ND 4.0

\section{REPOSITORY RECORD}

Balanov, Alexander G., Natalia B. Janson, V.V. Astakhov, and P.V.E. McClintock. 2019. "Role of Saddle Tori in the Mutual Synchronization of Periodic Oscillations". figshare. https://hdl.handle.net/2134/1748. 


\title{
Role of saddle tori in the mutual synchronization of periodic oscillations
}

\author{
A. G. Balanov, ${ }^{1,4, *}$ N. B. Janson, ${ }^{2}$ V. V. Astakhov, ${ }^{3}$ and P. V. E. McClintock ${ }^{4}$ \\ ${ }^{1}$ Institut für Theoretische Physik, Technische Universität Berlin, Hardenbergstraße 36, D-10623 Berlin, Germany \\ ${ }^{2}$ Department of Mathematical Sciences, Loughborough University, Loughborough, Leicestershire, LE11 3TU, United Kingdom \\ ${ }^{3}$ Department of Physics, Saratov State University, Astrakhanskaya 83, Saratov, 410026, Russia \\ ${ }^{4}$ Department of Physics, Lancaster University, Lancaster, LA1 4YB, United Kingdom
}

(Received 18 April 2005; published 19 August 2005)

\begin{abstract}
We show that in the mutual synchronization of periodic oscillators, besides an attracting torus, there is also a saddle torus that plays an equally important role. We demonstrate that the saddle and stable tori form an elegant structure, allowing for a variety of phenomena, both known and new, related to the origin and evolution of coexisting synchronous regimes (phase multistability).
\end{abstract}

DOI: 10.1103/PhysRevE.72.026214

PACS number(s): 05.45.Xt

\section{INTRODUCTION}

Synchronization is a fundamental phenomenon in the physics of oscillations. It manifests not only in physics, but also in biology, chemistry, and many other branches of science and engineering. It can involve coupled or forced periodic [1,2], chaotic [3-5], and even stochastic self-oscillators [6]. Synchronization implies an adjustment of the basic time scales of systems due to coupling between them. For periodic oscillations (the simplest case), synchronization can be attributed to certain bifurcations on an invariant ergodic torus. Two basic mechanisms of synchronization can be distinguished: phase (frequency) locking, which is associated with a saddle-node (fold) bifurcation of a saddle cycle and a stable limit cycle on the torus surface, and suppression of the natural dynamics, when an ergodic torus collapses into a limit cycle via a torus death (Neimark-Sacker) bifurcation $[2,7]$.

The dynamics of mutually coupled periodic oscillators is highly complex. In addition to synchronization, diverse phenomena such as amplitude death [8], chaotic oscillations [9], and phase multistability [10] can occur. Phase multistability means that, even for precisely the same set of control parameters, the interacting oscillators can be synchronized with different phase shifts depending on the initial conditions, which means that several synchronous attractors can coexist in their joint phase space. Phase multistability can even take place in the simple case of two mutually coupled Van der Pol oscillators [10].

Although the general mechanisms for mutual synchronization have been intensively studied, there remain a number of unsolved fundamental problems. In particular, it is known that inside the locking region the coexisting limit cycles usually live on the surface of the same torus, their basins of attraction being separated by the manifolds of saddle cycles

\footnotetext{
*Corresponding author. Electronic addresses: balanov@physik.tuberlin.de, n.b.janson@lboro.ac.uk, astakhov@chaos.ssu.runnet.ru, p.v.e.mcclintock@lancaster.ac.uk
}

lying on the same torus. It is equally well established that, as one leaves the locking region and enters the suppression region, the saddle cycles with their delimiting manifolds disappear through a bifurcation. However, phase multistability continues to exist. So several questions immediately arise: As one crosses the boundary between locking and suppression, how does the structure of phase space change? What bifurcations accompany this change? What separates the basins of attraction of the coexisting limit cycles?

Note that the coexistence of different limit sets inside the synchronization region is a very general phenomenon that is observed in many interacting systems with diverse forms of coupling [11-16], so that these are important questions of considerable general interest. Most works on phase multistability study the case of weak linear coupling, for which synchronzation is realized via frequency/phase locking; it remains unclear how the phase space structure evolves in the cases of large coupling and/or nonlinear coupling.

In this paper we study the simplest representative example of two periodic mutually coupled oscillators with either linear or nonlinear coupling, which describes the general case of coexisting limit cycles and of associated bifurcational transitions. We reveal the phase space structure of the system under study, allowing us to explain the phenomena mentioned above. In Sec. II we introduce the system to be studied and identify explicitly the questions to which we seeks answers. In Sec. III we discuss the form of phase space necessary to account for the observed phenomena and thus provide answers to the questions posed. In order to validate the hypothesized phase space structure, it is analyzed in detail and subjected to a variety of tests in Sec. IV. It is shown to be self-consistent and to meet all requirements. In Sec. V we check the generality of our results considering other example of coupled oscillators. Finally, in Sec. VI, the work is summarized and conclusions are drawn.

\section{SYSTEM UNDER STUDY AND QUESTIONS POSED}

As a paradigm for a pair of coupled, periodic, selfsustained oscillators, we consider two mutually coupled Van der Pol systems: 


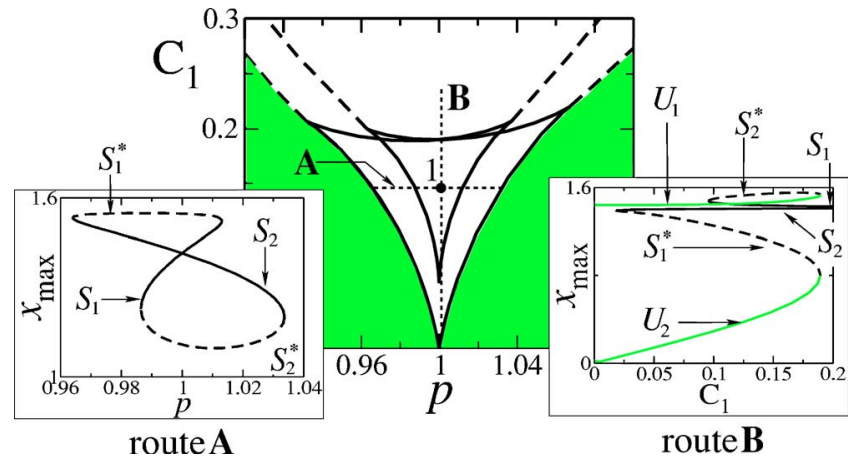

FIG. 1. (Color online) Central figure: bifurcation diagram showing the synchronization region for linear coupling, on the $p-C_{1}$ (detuning-coupling strength) plane. In the shaded areas there are no 1:1 synchronous solutions. The full lines are fold bifurcations, and the heavy dashed lines are Neimark-Sacker bifurcations leading to torus birth. The dotted lines show the two routes A and B across the diagram that are illustrated in the insets. The filled black circle 1 marks the parameter values for which the structure of phase space is shown in Fig. 5(b). Inset A shows how the positions of the maxima $x_{\text {max }}$ of the stable $S_{1,2}$ and single-saddle $S_{1,2}^{*}$ cycles depend on $p$ for $C_{1}=0.15$. Inset B shows how $x_{\max }$ of all six cycles depend on $C_{1}$ for $p=1.002$. In insets full lines represent $S_{1,2}$, dashed lines $S_{1,2}^{*}$, and grey lines the double-saddle cycles $U_{1,2}$.

$$
\begin{gathered}
\dot{x}_{1,2}=y_{1,2}, \\
\dot{y}_{1,2}=\left(\varepsilon_{1,2}-x_{1,2}^{2}\right) y_{1,2}-\omega_{1,2}^{2} x_{1,2}+C_{1}\left(x_{2,1}-x_{1,2}\right) \\
+C_{2}\left(x_{2,1}-x_{1,2}\right)^{2} .
\end{gathered}
$$

Here, $\varepsilon_{1,2}$ are nonlinearity parameters, $\omega_{1}=\omega$ and $\omega_{2}=p \omega$ are eigenfrequencies, where $p$ introduces detuning between the systems, and $C_{1}, C_{2}$ are the strengths of the linear and of quadratic couplings, respectively.

We initiate our studies by considering the simplest linear coupling $\left(C_{1} \neq 0, C_{2}=0\right)$ leading to the coexisting limit sets and associated bifurcations of interest. However, in order to estimate how a more complex coupling influences the coexisting regimes and their evolution, we also consider a nonlinear interaction where the coupling function includes a quadratic term. Although in what follows we fix equal nonlinearities $\varepsilon_{1}=\varepsilon_{2}=0.5$, all results reported below were also reproduced for unequal nonlinearities, $\varepsilon_{1}=0.5$ and $\varepsilon_{2}=0.52$. We choose $\omega=1$, and change $p$ around 1 in order to study the basic $1: 1$ synchronization region. For these parameter values there are six cycles involved: two stable cycles that we further refer to as $S_{1,2}$, two saddle cycles (with one unstable direction) $S_{1,2}^{*}$, and two "double-saddle" cycles (with two unstable directions) $U_{1,2}$. These cycles can undergo two types of bifurcation: fold while merging in pairs $\left(S_{1,2}\right.$ with $S_{1,2}^{*}$, or $S_{1,2}^{*}$ with $\left.U_{1,2}\right)$, or torus birth ( $S_{1,2}$ only).

In the bifurcation diagrams shown in Figs. 1-3, shaded regions mark the absence of 1:1 synchronization and will not be considered further in the present work.

\section{A. Linear coupling and multistability}

First, we study linear mutual coupling by setting $C_{2}=0$. In the $p-C_{1}$ parameter plane the region of phase locking (the
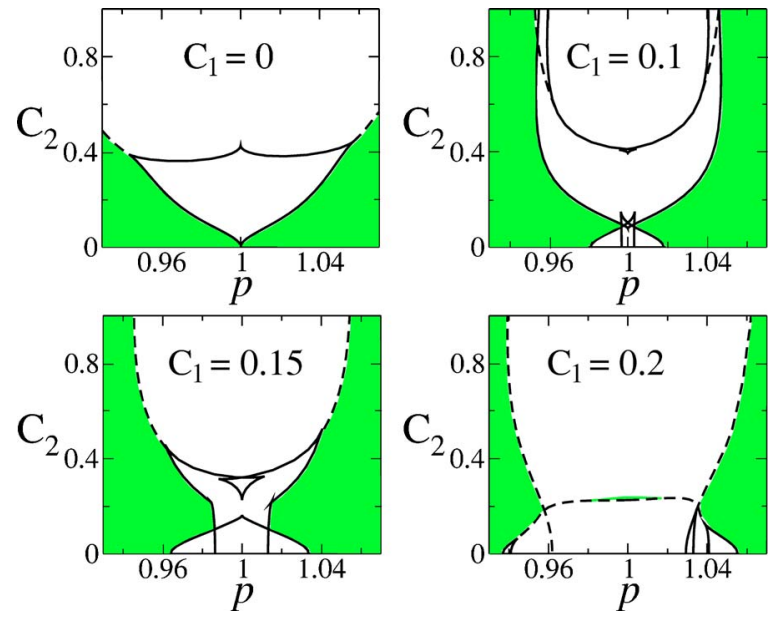

FIG. 2. (Color online) Bifurcation diagrams in the $p-C_{2}$ parameter plane for four different values of $C_{1}$. In the shaded areas there are no 1:1 synchronous solutions. Full black lines are fold bifurcations; dashed lines show Neimark-Sacker bifurcation; full grey line for $C_{1}=0.2$ (see also Fig. 3) shows fold bifurcations for stable and saddle tori.

main part of Fig. 1) [17] is outlined by full lines on which fold bifurcations between different pairs of cycles occur. Dashed lines mark torus birth bifurcation and enclose the region of suppression. At very small $C_{1}$, the locking region looks like a classical Arnol'd tongue. However, at larger $C_{1}$ another narrower "tongue" can be found, embedded within the first one.

Within the smaller tongue $S_{1}$ and $S_{2}$ coexist on the surface of the same stable torus, separated by $S_{1}^{*}$ and $S_{2}^{*}$, while $U_{1,2}$ live in the neighborhood. To illustrate how cycles appear/ disappear within and at the boundaries of the phase-locking region, we follow routes $\mathbf{A}$ and $\mathbf{B}$ in Fig. 1, as marked by the dotted lines. Insets show evolution of cycles maxima $x_{\max }$ along the routes. Along route $\mathbf{A}$ (the left-hand inset of Fig. $1), S_{1,2}$ and $S_{1,2}^{*}$ merge or appear in pairs, always on the same

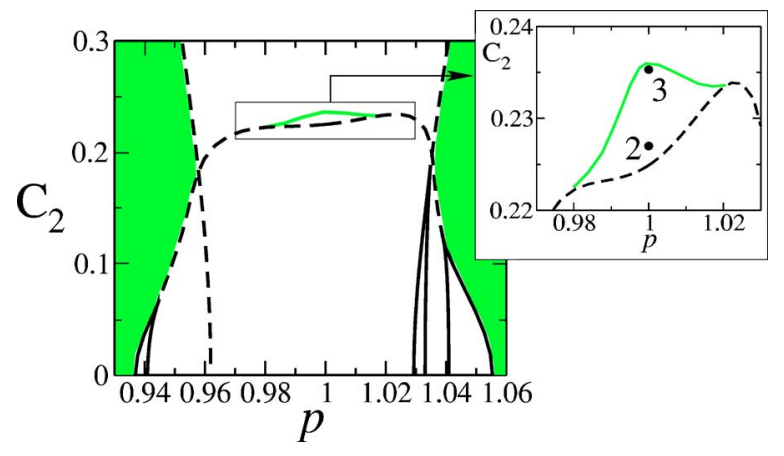

FIG. 3. (Color online) Bifurcation diagram on the $p-C_{2}$ plane for $C_{1}=0.2$. In the shaded areas there are no 1:1 synchronous solutions. Full black lines are fold bifurcations for the pairs of cycles $S_{1,2}$ and $S_{1,2}^{*}$, and $S_{1,2}^{*}$ and $U_{1,2}$. Dashed lines are Neimark-Sacker bifurcations for the cycles $S_{1,2}$. A full grey (green online) line is the fold bifurcation for a saddle torus $T^{*}$ and a stable torus $T_{2}$ born from $S_{2}$. In the region bounded by the dashed line from below and by the full grey line from above (see the inset), a stable cycle $S_{1}$, and a stable torus $T_{2}$ coexist. 
torus surface. $U_{1,2}$ that do not undergo bifurcations exist throughout the route (not shown). Along route $\mathbf{B}$ (the righthand inset of Fig. 1) all six cycles undergo fold bifurcations. Above the upper border of the phase-locking region at $C_{1}$ $\approx 0.192$, there are only two cycles $S_{1,2}$ left, both stable.

It is evident from the right-hand inset of Fig. 1 that, for $C_{1}=0.2, p=1.002$ (i.e., inside the suppression region), two stable limit cycles $S_{1,2}$ coexist in phase space. However, there are no saddle cycles $S_{1,2}^{*}$ whose stable manifolds could separate the basins of attraction. So the following question arises: What separates the basins of attraction here?

\section{B. Nonlinear coupling and disappearance of a stable torus}

Now let us take account of nonlinear coupling. We have two coupling parameters $C_{1}$ and $C_{2}$, and one detuning parameter $p$, so the control parameter space of interest becomes three dimensional. Consequently, we will have bifurcation surfaces rather than lines. Because it is difficult to visualize surfaces of such intricate shape intertwining in the threedimensional (3-D) parameter space, we show four representative sections in the plane $p-C_{2}$ at four fixed values of $C_{1}$ (Fig. 2). One can see that at $C_{1}=0$, i.e., when the coupling is quadratic without a linear component, the synchronization tongue possesses the classical shape that exists in a harmonically forced periodic oscillator. It is even simpler than for purely linear coupling (cf. Fig. 1) and has single-leaf structure, implying the absence of phase multistability. As $C_{1}$ increases, however, the bifurcation diagram becomes more involved, with new bifurcation lines appearing, crossing each other, and merging. Also, in some parts of the diagrams two stable objects evidently coexist in the phase space, with the diagrams becoming multileaf. In each of Figs. 2(a)-2(d), an increase of $C_{2}$ eventually leads to the disappearance of one of the coexisting periodic regimes as a result of a certain bifurcation, so that the diagram becomes single leaf.

Consider in more detail the lower part of the diagram for $C_{1}=0.2$ (Fig. 3). The left and right-hand borders of the synchronization region are formed by lines of fold bifurcations of pairs of cycles $S_{1,2}$ with $S_{1,2}^{*}$, and of $S_{1,2}^{*}$ with $U_{1,2}$, as shown by full lines. On dashed lines, stable cycles $S_{1,2}$ lose their stability via Neimark-Sacker bifurcations. The latter can either be supercritical, leading to the birth of stable tori, or subcritical not resulting in a torus birth.

Note that the point $C_{1}=0.2, C_{2}=0, p=1.002$ in Figs. 2 and 3 is in fact identical with point $C_{1}=0.2, p=1.002$ in Fig. 1 . Thus the same limit cycles $S_{1,2}$ coexist here as those illustrated on the right-hand inset of Fig. 1 at $C_{1}=0.2$.

In Fig. 3, on the almost horizontal segment of dashed line, the stable cycle $S_{2}$ undergoes a Neimark-Sacker bifurcation. If after crossing the dashed line one finds oneself in the region bounded by a dashed line from below, and by full grey (green online) from above (see the inset of Fig. 3), a stable torus $T_{2}$ is born, and the cycle $S_{2}$ becomes a double-saddle cycle (we mark its instability by an overtilde: $\widetilde{S}_{2}$ ). An interesting component of this bifurcation diagram is the full grey (green online) line on which $T_{2}$ disappears via a bifurcation. Since the latter is not of a cycle, but of a torus, the corresponding line was obtained not by a continuation algorithm,
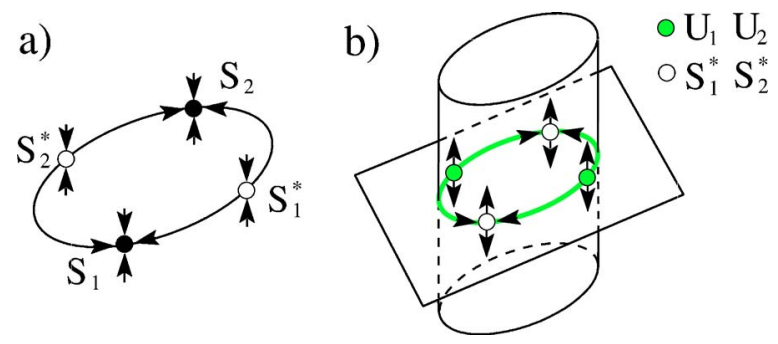

FIG. 4. (Color online) Sketches of Poincare sections of (a) the attracting resonant torus $T$, (b) the saddle resonant torus $T^{*}$ shown as a closed grey (green online) curve, and its manifolds shown as surfaces. The circles indicate cycles: filled ones represent the stable cycles $S_{1,2}$; open ones represent the single-saddle cycles $S_{1,2}^{*}$; and grey (green online) ones represent the double-saddle cycles $U_{1,2}$.

but by observing phase portraits and marking the parameter values at which $T_{2}$ vanished. Remarkably, there is no sign of torus distortion or of the quasiperiod tending to infinity before the bifurcation, as usually accompany homoclinic bifurcations: the torus vanishes while remaining smooth and of finite size. So the question here is as follows: Why does the torus disappear?

\section{HYPOTHESIZED STRUCTURE OF THE PHASE SPACE}

To answer the questions posed in Sec. II, we have to reveal the structure of phase space, and to show that it allows for all observed bifurcations of limit cycles and provides a separating surface for the coexisting stable cycles in the region of suppression. This structure should also explain the sudden disappearance of the smooth torus $T_{2}$.

The main facts to be accounted for are as follows.

(1) Both stable cycles $S_{1,2}$, together with both singlesaddle cycles $S_{1,2}^{*}$ lie on the same torus, as illustrated by the left-hand inset of Fig. 1.

(2) As partly illustrated by insets of Fig. 1, any singlesaddle cycle $S_{1,2}^{*}$ is able to merge, either with any stable cycle $S_{1,2}$, or with any double-saddle cycle $U_{1,2}$.

From facts (1) and (2) we hypothesise that, inside the central part of locking region of Fig. 1 containing point (1), there exist two different tori in the phase space, in contrast to the single torus in the case of a forced 2-dimensional system. Of these, the first is an attracting resonant torus whose dimension is 2 and whose surface is formed by the 2-dimensional unstable manifolds of the single-saddle cycles $S_{1,2}^{*}$ that close on stable cycles $S_{1,2}$. A sketch of its Poincaré section is shown in Fig. 4(a) by the full black closed curve; circles mark the positions of cycles. This resonant torus is similar to that which exists in a forced system, but now, instead of one pair of cycles, it has two pairs of cycles lying on it.

The second torus is a saddle resonant torus, also of dimension 2. In the Poincaré section a two-dimensional saddle torus will look like a saddle cycle, and its manifolds will look like those of a saddle cycle. A section of a resonant saddle torus is sketched in Fig. 4(b) together with its manifolds. In the full phase space, a saddle torus is the intersec- 

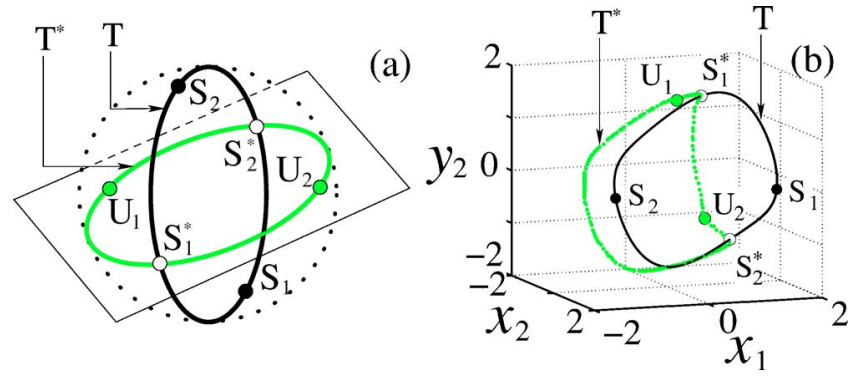

FIG. 5. (Color online) Poincaré sections of objects in the phase space. (a) Hypothesized structure of phase space inside the region where phase locking of the mutually coupled periodic oscillators occurs. A dotted line shows the "sphere," which in fact corresponds to the cylinder in Fig. 4(b) that is closed from below and above. (b) A numerically revealed structure of the phase space at parameter values corresponding to the point labeled 1 in Fig. 1 . The circles indicate cycles: filled ones are the stable cycles $S_{1,2}$; open ones are the single-saddle cycles $S_{1,2}^{*}$; grey (green online) are the doublesaddle cycles $U_{1,2}$. The full closed curves show tori: the black ones are stable $T$, and grey (green online) ones are saddle $T^{*}$. For details, see the text.

tion of two three-dimensional manifolds. We note that the minimum dimension of the phase space into which a threedimensional manifold can be embedded, and thus where a saddle torus could exist, is four dimensional-which is indeed the dimension of the system, Eqs. (1), under study.

The manifolds that cross to form a saddle torus play a very important role, and they need to be revealed. However, their dimension is 3 , so that they cannot be visualized though projection from the four-dimensional to the threedimensional space. For this reason, throughout this paper, instead of showing projections of the objects involved, we will show their Poincaré sections. Thus cycles will turn into points, tori into closed curves, and three-dimensional manifolds into surfaces in three-dimensional space.

We hypothesize that the two tori intersect at the singlesaddle cycles $S_{1,2}^{*}$ as shown in Fig. 5(a).

(3) Given facts 1 and 2, both tori should lie on the same closed hypersurface ("sphere"). The latter is the unstable manifold of the saddle torus sketched as the cylinder in Fig. 4(b) which, instead of going to infinity, should be closed from below and from above. This "sphere" would then be a central manifold for the Neimark-Sacker bifurcation of the stable cycle $S_{2}$ on the dashed line in Fig. 3 .

The Poincaré section of the hypothesised structure in the three-dimensional space is sketched in Fig. 5(a). Here, cycles are given by circles: black $-S_{1,2}$, white $-S_{1,2}^{*}$, grey (green online) $-U_{1,2}$. Full closed curves show tori. Surfaces show manifolds of the saddle torus: horizontal plane-stable, and sphere-unstable manifolds, respectively.

If the structure shown in Fig. 5(a) is correct, the stable manifold of the saddle torus shown as a plane is the soughtafter surface separating the basins of the two coexisting cycles $S_{1,2}$. Although the proposed structure is self-consistent and would provide an elegant answer to the above questions, its existence needs to be verified. The best way to do this is to try to visualize the structure numerically. However, at the moment there seems to be no numerical method available that would allow one to visualize a saddle torus. We therefore require a way to plot the inferred saddle torus using what we know about the system and exploiting all necessary implications of the hypothesized structure's existence. We address this problem in the next section.

\section{VERIFYING THE HYPOTHESIZED STRUCTURE OF THE PHASE SPACE}

\section{A. Structure at linear coupling with multistability}

First, we note that our hypothesis assumes two pairs of cycles $S_{1,2}$ and $S_{1,2}^{*}$ lying on the same closed curve (the resonant torus) $T$. An indication of this can be seen in Fig. 1 (inset A) showing that with the change in $p$ all four cycles move along the same closed curve. However, the assumption must also be verified for fixed system parameters. A resonant torus can be visualized numerically with the help of an existing technique [18] that involves surrounding the torus by a closed surface and following its evolution in time. Since $T$ is an attracting structure as a whole, and despite the real attractors being cycles $S_{1,2}$ lying on it, the closed surface will tend eventually to coincide with the torus. Performing this calculation for $C_{2}=0, p=1.002$, and $C_{1}=0.15$ in Eq. (1) (point 1 in Fig. 1), we obtained a closed curve in the Poincare section (the black curve in Fig. 5(b)) representing the torus $T$ sought. As an independent test of validity of this torus, we plot the cycles $S_{1,2}$ and $S_{1,2}^{*}$ in the same figure to show that they do indeed lie on the closed curve found.

Second, we note that, to enable trajectories from inside the sphere to be attracted to its surface, a repelling object should exist inside it; and, indeed, it can be easily shown that there is a repelling fixed point at the origin. If time is reversed, a repeller becomes an attractor; and, if the hypothesized sphere exists, it will become the boundary between the basins of attraction of the fixed point and of infinity. We were thus able to compute the basin of attraction of the fixed point in reversed time for the above parameters, and estimated its boundary numerically. This indeed appeared to be a closed surface topologically equivalent to a sphere (not shown, to avoid overloading the figures).

Third, if the hypothesized picture is true, both the stable torus $T$ and double-saddle cycles $U_{1,2}$ should lie on the sphere. $T$ should divide the sphere into two halves, each containing one of the double-saddle cycles, a conclusion that was verified within our numerical accuracy.

Fourth, the manifold sketched in Fig. 5(a) as a plane separating the basins of attraction of coexisting limit cycles $S_{1}$ and $S_{2}$, can be calculated in a similar way to that used for the sphere, through a calculation of the basins. The surface thus found (again, not shown) was indeed not closed, at least within the relevant part of phase space.

Fifth, if the saddle torus exists, it should be a closed curve at which the surfaces found above intersect each other. We have established that the sphere and the plane do indeed intersect, and have estimated their points of intersection numerically, yielding the closed curve shown in Fig. 5(b) by grey (green online), that is supposedly the saddle torus $T^{*}$ being sought (cf. Fig. 5(a)). 
Finally, the saddle torus $T^{*}$ should intersect with the stable torus $T$ at cycles $S_{1,2}^{*}$, and $U_{1,2}$ should lie on its surface as well. This too appears to be true within numerical accuracy (compare Fig. 5(b) with Fig. 5(a)). Because the calculation of basins of attraction does not require knowledge of the positions of cycles $S_{1,2}^{*}$ and $U_{1,2}$, the fact that they were found to be in the expected places provides another independent argument supporting the validity of the structure under consideration.

Our hypothesized structure of phase space in the central part of the locking region for two mutually coupled periodic oscillators is thus shown to be valid. This structure provides the answer to the question: What separates the basins of attraction of coexisting limit cycles $S_{1,2}$ in the region of suppression lying above all the full lines in the upper part of the bifurcation diagram of Fig. 1? In this region, the saddle cycles $S_{1,2}^{*}$ do not exist, having merged with the doublesaddle cycles $U_{1,2}$ via fold bifurcations. However, the saddle torus on which all the above cycles lived continues to exist as an ergodic one, and thus provides the required threedimensional stable manifold that serves as a delimiting surface.

\section{B. Structure at nonlinear coupling and disappearance of a stable torus}

Next, we consider the abrupt disappearance of the newly born torus $T_{2}$ under nonlinear coupling, as the grey (green online) line of Fig. 3 is crossed from below to above. Below the dashed line lies the region of suppression, with a phase space structure that is qualitatively the same as in the suppression region of Fig. 1. This means that in the phase space there coexist two stable cycles $S_{1,2}$, and a saddle torus that is now ergodic. When the torus $T_{2}$ is born from $S_{2}$ on the dashed line, the phase space structure must look like that sketched in Fig. 6(a). Note that $T_{2}$ must also lie on the "sphere" since it is the central manifold of torus birth bifurcation.

As parameter $C_{2}$ is gradually increased above the value of torus birth bifurcation, the torus diameter increases. However, being bounded to lie on the sphere, the only opportunity for it to grow is to move toward the equator, i.e., toward the saddle torus $T^{*}$. The inevitable consequence is that, eventually, $T_{2}$ must collide with $T^{*}$ and disappear, which would imply a fold bifurcation. After that, the only attractor of the system would be the stable cycle $S_{1}$.

This suggestion was tested by use of methods similar to those resulting in Fig. 5(b). For each of two sets of parameters inside the region where the cycle $S_{1}$ and torus $T_{2}$ coexist, marked as points 2 and 3 in Fig. 3, the two tori were obtained numerically. In Fig. 6(b) the phase space is illustrated for point 2, and in Fig. 6(c) for point 3. It is clearly seen that, immediately after the torus birth bifurcation (Fig. 6(b)), the new torus $T_{2}$ is small and is situated relatively far from the saddle torus $T^{*}$. Also, it does belong to the sphere that is not illustrated here. However, close to the parameter value at which $T_{2}$ disappears (Fig. 6(c)), both tori are very close to each other, almost coinciding in shape and size. These figures support our hypothesis of a fold bifurcation of
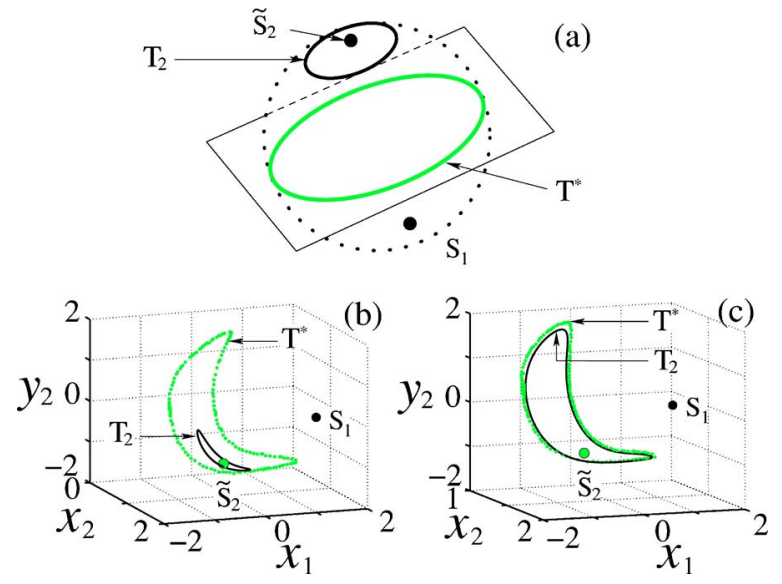

FIG. 6. (Color online) Poincare sections of objects in the phase space. (a) A hypothesized structure of phase space inside the suppression regime for the mutually coupled periodic oscillators, in particular, inside the region containing points 2 and 3 in the inset of Fig. 3, where a stable cycle coexists with a stable torus. (b) and (c) A numerically revealed structure of the phase space at parameter values corresponding to points 2 and 3 in Fig. 3, respectively. Designations are as in Fig. 5. The full black line here shows the newly born stable torus $T_{2}$.

the two tori and thus provide the mechanism by which the stable torus disappears.

\section{GENERALITY CHECK: PHASE SPACE STRUCTURE IN A DIFFERENT SYSTEM}

After having revealed the structure of the phase space for two coupled Van der Pol oscillators, each being a paradigm for periodic self-sustained oscillators, we are naturally interested to seek evidence as to whether this structure is general. So we consider another system of two coupled periodic selfoscillators, namely, two simplified FitzHugh-Nagumo systems in their oscillatory regimes, each of the form

$$
\begin{gathered}
\dot{x}_{1,2}=x_{1,2}-x_{1,2}^{3} / 3-y_{1,2}, \\
\dot{y}_{1,2}=x_{1,2}+a+C\left(y_{2,1}-y_{1,2}\right),
\end{gathered}
$$

for control parameter values $a=0.1, C=0.17$. Here we consider identical oscillators to ensure that they are $1: 1$ synchronized. At our chosen value of $C=0.17$ they are in fact phase locked, as can be verified by a bifurcation analysis.

All stages of the computation were carried out in close analogy with the coupled Van der Pol systems, Eqs. (1), described in Sec. IV B. The final configuration of objects found in the phase space is as shown in Fig. 7. One can see immediately that the objects themselves, and their configuration, are just as hypothesized (Fig. 5(a)) and are essentially the same as in the coupled Van der Pol systems (Fig. 5(b)).

\section{CONCLUSIONS}

In conclusion, we have revealed the structure of the joint phase space of two mutually coupled periodic Van der Pol oscillators within the synchronization regime, thereby en- 


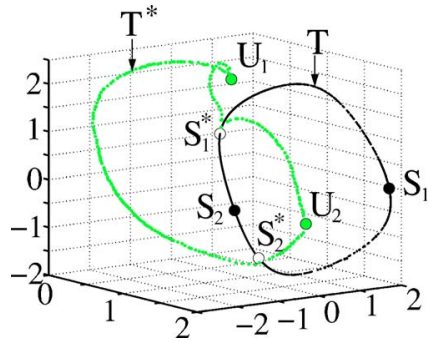

FIG. 7. (Color online) Poincaré sections of objects in the phase space of two coupled FitzHugh-Nagumo systems, Eqs. (2). Designations are as in Fig. 5.

abling us to answer the questions posed in the Introduction and Sec. II. We have shown that, quite generally, there are two tori lying on the same closed manifold, intersecting to form an elegant structure. All evolutions of synchronous attractors are closely associated with bifurcations of solutions belonging to either saddle tori, or stable tori, or both, or of the tori themselves. As discussed in Sec. V, the same phase space structure was also revealed in two coupled FitzHughNagumo systems in their oscillatory regime. A deeper understanding of the physics of such interacting oscillators, and their complex mutual phase space structure, could facilitate the manipulation of dynamical systems with phase multistability in a variety of practical applications, e.g., in biology and medicine, where phase multistability is often of vital importance $[4,11]$.

In addition, we have introduced a method of visualizing saddle tori that could prove useful in the wide variety of problems where such objects appear.

\section{ACKNOWLEDGMENTS}

The work was supported by Leverhulme Trust, the U.S. Civilian Research Development Foundation (Grant No. REC 006), and the Engineering and Physical Sciences Research Council (UK).
[1] V. I Arnold, Geometrical Methods in the Theory of Ordinary Differential Equations (Springer-Verlag, New York, 1983).

[2] P. S. Landa, Self-Oscillations in Systems with Finite numbers of Degrees of Freedom (Nauka, Moscow, 1980) (in Russian).

[3] A. Pikovsky, M. Rosenblum, and J. Kurths, Synchronization: A Universal Concept in Nonlinear Science (Cambridge University Press, Cambridge, 2001).

[4] E. Mosekilde, Yu. Maistrenko, and D. Postnov, Chaotic Synchronization. Applications to Living Systems (World Scientific, Singapore, 2002), Series A, Vol. 42.

[5] S. Boccaletti, J. Kurths, G. Osipov, D. L. Valladares, and C. S. Zhou, Phys. Rep. 366, 1 (2002).

[6] V. S. Anishchenko, V. V. Astakhov, A. B. Neiman, T. E. Vadivasova, and L. Schimansky-Geier, Nonlinear Dynamics of Chaotic and Stochastic Systems (Springer-Verlag, Berlin, 2002).

[7] J. Guckenheimer and P. Holmes, Nonlinear Oscillations, Dynamical Systems, and Bifurcations of Vector Fields (SpringerVerlag, New York, 1986).

[8] K. Bar-Eli, Physica D 14, 242 (1985).

[9] V. S. Afraimovich and L. P Shilnikov, Am. Math. Soc. Transl. 149, 201 (1991).
[10] D. G. Aronson, E. J. Doedel, and H. G. Othmer, Physica D 25, 20 (1987).

[11] E. Mosekilde, D. E. Postnov, O. V. Sosnovtseva, Prog. Theor. Phys. Suppl. 150, 147 (2003).

[12] D. E. Postnov, T. E. Vadivasova, O. V. Sosnovtseva, A. G. Balanov, and E. Mosekilde, Chaos 9, 227 (1999).

[13] S. H. Park, S. Kim, H.-B. Pyo, and S. Lee, Phys. Rev. E 60, 2177 (1999).

[14] V. V. Astakhov, B. P. Bezruchko, E. N. Estrova, and E. P. Seleznev, Sov. Phys. Tech. Phys. 35, 1122 (1990).

[15] J. Rasmussen, E. Mosekilde, and Ch. Reick, Math. Comput. Simul. 40, 247 (1996).

[16] T. E. Vadivasova, O. V. Sosnovtseva, A. G. Balanov, and V. V. Astakhov, Discrete Dyn. Nat. Soc. 4, 231 (2000).

[17] All bifurcation diagrams in this Letter were obtained using an AUTO-2000 package: E. Doedel, R. C. Paffenroth, T. F. Fairgrieve, Y. A. Kuznetsov, B. E. Oldeman, B. Sandstede, and X. Wang, "AUTO2000: Continuation and bifurcation software for ordinary differential equations (with HOMCONT)," Technical report, Concordia University, 2002.

[18] I. G. Kevrikidis, R. Aris, and L. D. Schmidt, Physica D 23, 391 (1986). 